Negar Chabi, Oliver Beuing, Bernhard Preim and Sylvia Saalfeld*

\title{
Automatic stent and catheter marker detection in $X$-ray fluoroscopy using adaptive thresholding and classification
}

https://doi.org/10.1515/cdbme-2020-0006

\begin{abstract}
In this study, we propose a method for marker detection in X-ray fluoroscopy sequences based on adaptive thresholding and classification. Adaptive thresholding yields multiple marker candidates. To remove non-marker areas, 24 specific features are extracted from each extracted patch and four supervised classifiers are trained to differentiate non-marker areas from marker areas. Quantitative evaluation was carried out to assess different classifier performance by calculating accuracy, sensitivity, specificity and precision. SVM outperforms other classifiers based on the mean value for accuracy, specificity and precision with $81.56,91.94$ and $84.21 \%$, respectively.
\end{abstract}

Keywords: binary classification; feature extraction; flow diverter; opaque-marker; stent; X-ray fluoroscopy.

\section{Introduction}

Intracranial stenting is one of the minimally-invasive therapies for neurovascular pathologies such as intracranial aneurysms and is accomplished via continuous $\mathrm{X}$-ray imaging. Intracranial stents and flow diverters are small flexible mesh tube devices. Precise real-time device placement depends on the proper intra-operative visualization of the stent. Although radio-opaque stent markers are visible in fluoroscopy, they are as small as possible and their detection is challenging.

Sabrina et al. [1] proposed a technique to detect stentgraft markers to support the physicians with the stent-graft deployment in intra-operative fluoroscopic images. Fast

*Corresponding author: Sylvia Saalfeld, Department of Simulation and Graphics, Forschungscampus STIMULATE, OvG-University, Magdeburg, Germany, E-mail: sylvia.saalfeld@ovgu.de Negar Chabi and Bernhard Preim, Department of Simulation and Graphics, OvG-University, Magdeburg, Germany,

E-mail: negar.chabi@ovgu.de (N. Chabi),

bernhard.preim@ovgu.de (B. Preim)

Oliver Beuing, Department of Radiology, Ameos Hospital Bernburg, Bernburg, Germany, E-mail: obeu.rad@bernburg.ameos.de radial symmetry transform following an ellipse or line fitting approach was used to detect a group of markers.

Movassaghi et al. [2] proposed a 3D representation of the deployed stent during a percutaneous transluminal coronary angioplasty procedure. Markers on the balloon catheter were utilized for motion compensation. Here the markers are clearly visible because of the high absorption coefficients and are detected using a scale-space algorithm.

Bismuth et al. [3] presented a technique to improve the stent visualization on an X-ray image sequence. Stent motion is inferred based on the motion of balloon marker balls as well as a guide wire. Potential marker balls are detected by local minima selection and a priori knowledge.

Wang et al. [4] proposed a fully balloon marker pair tracking and guide wire localization to employ 3D motion compensation and stent reconstruction. Marker candidates were detected using a probabilistic boosting tree classifier.

Many studies used blob-like structure detectors to detect markers [4-6]. Blob analysis actually refers to methods aiming at detecting regions with different characteristics, such as brightness or color, compared to their neighborhood.

Many approaches for stent marker detection are not applicable to intracranial stents because of different properties. Most studies focus on coronary stent enhancement (e.g., balloon-mounted stent with a couple of radioopaque markers connected by a guide wire [3-6]). This specific geometry is used to remove false positive candidate markers [6]. However, we are dealing with a type of intracranial stent, e.g., self-expanding stents with a different number of radio-opaque markers, which are not connected by a specific landmark.

In this paper, we focus on the X-ray opaque marker detection in intracranial fluoroscopic images. We have implemented a multi-step marker detection algorithm for fluoroscopy sequences based on an adaptive thresholding technique and binary classification.

We use blob analysis as feeding features to train classifiers. Different classifiers include linear discriminant analysis classifier (LDA), naïve Bayesian classifier (NB), k-nearest neighbors (KNN) and support vector machine (SVM) which will be described in the next section. 


\section{Methodology}

We develop an algorithm to detect radio-opaque markers located on intracranial stent and catheter.

\section{Data acquisition}

Seven clinical X-ray fluoroscopy sequences were used in this study. Images were acquired at the Institute for Neuroradiology, Magdeburg, Germany with an Artis Q (Siemens Healthineers, Forchheim, Germany). The image size is $512 \times 512$ pixels. Frame rates are between 8 and $30 \mathrm{fps}$.

Different types of stents were used for treatment, for example the DERIVO flow diverter (ACANDIS GmbH Co. KG, Pforzheim, Germany), a self-expanding stent with $15-80 \mathrm{~mm}$ length in the vessel and applicable for $2.5-6 \mathrm{~mm}$ vessel diameter.

\section{Pre-processing and patch extraction}

To enhance the visibility of the stent markers, a simple preprocessing technique was employed which is described in our previous work [7]. Afterward, adaptive thresholding [8] was employed to identify marker areas. The sensitivity is a parameter ranged in [0-1]. Higher sensitivity equals a higher number of detected regions as foreground. It was empirically selected in [0.4-0.5] for different frames. Patch extraction includes detecting components connecting in the diagonal, vertical, and horizontal direction. Then, corresponding patches were extracted from the original frame and its corresponding pre-processed frame. Patch size is empirically selected $15 \times 15$ to cover even very big markers such as catheter markers. Since other structures, which are not of interest, such as guide wire or catheter, are also detected as foreground, eccentricity and area were used to get rid of them. Detected regions with higheccentricity were removed using 0.98 as a threshold. This threshold was selected empirically. Regions larger than a threshold (e.g., coil) were removed. The threshold was empirically chosen based on the marker size (bigger than the maximum marker size), estimated radiographic magnification factor and image pixel spacing.

\section{Feature extraction}

Due to the blob-like appearance of the desired regions, we employ different blob analysis algorithms to train different classifiers against false positives. For each patch with a size of $15 \times 15$, we extract 22 features, which include minimum, average, and standard deviation, as well as the following features.

Laplacian of Gaussian (LoG) computes the second derivative of a function and detects abruptly changing regions. The filter size is empirically selected 15 and the standard deviation is the default value. Then, mean and standard deviation of LoG were fed in the classifiers.

Difference of Gaussian (DoG) can be used to detect edges. The mean and standard deviation of DoG were computed. The standard deviation ratio between two Gaussian was selected 2, and the filter sizes were selected 10 times of their standard deviation. These values were empirically chosen.

Determinant of Hessian (DoH) detects blobs in an image. The Hessian matrix contains the second partial derivatives of a function. DoH was calculated in two different ways: one is the second derivative of the input and the other one is to filter the input with the second derivative of a Gaussian filter with standard deviation at its default value [9]. Then, mean and standard deviation of the computed DoH matrix for patches extracted from both preprocessed and original frame are considered as input features.

\section{Classification}

Different classifiers including k-nearest neighbor (KNN), naive Bayesian classifier (NB), support vector machine (SVM) and linear discriminant analysis (LDA) classifier were trained and used to differentiate patches with and without markers.

\section{Experiments and results}

The framework for our approach is illustrated in Figure 1. For training and test, we used four and three clinical fluoroscopy sequences, respectively. To detect true X-ray markers, classifiers are trained to discriminate marker regions from non-marker regions. Features were extracted from 112 patches of some arbitrary frames of 4 clinical fluoroscopy sequences to train the classifiers. Different classification techniques were employed to differentiate patches. SVM classification was performed by standardizing the features, and sequential minimal optimization (SMO) was chosen as an optimization routine. The radial basis function was selected as a kernel function. For the KNN classifier, the distance metric was Euclidean distance and the number of nearest neighbor (K) was 1. Both LDA 


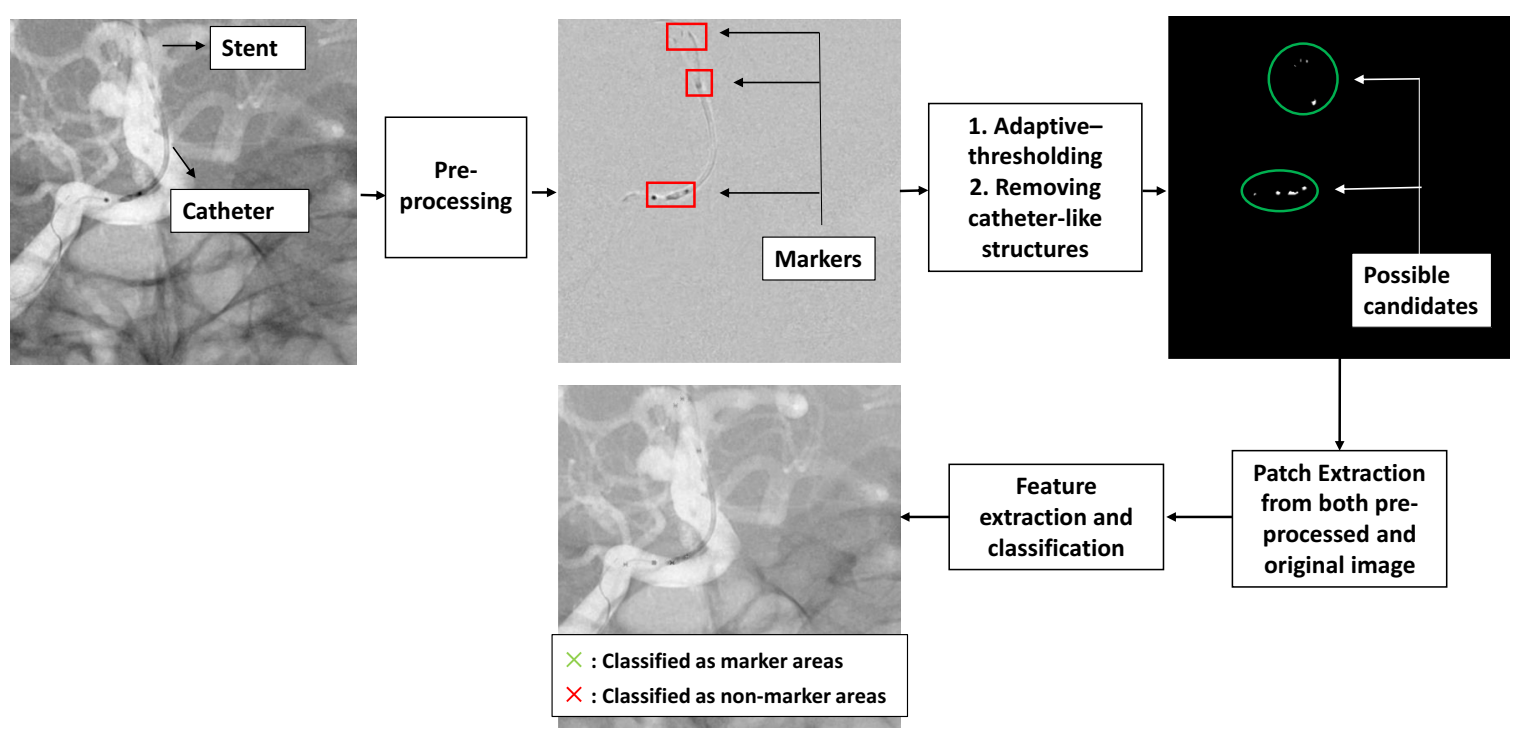

Figure 1: Workflow for the proposed approach.

and NB classifiers were trained in their default mode in MATLAB (MathWorks, Natick, USA). To evaluate the performance of the approach, 1948 test patches were extracted from various frames of 3 different fluoroscopy sequences. The number of true positive (TP), true negative (TN), false positive (FP) and false negative (FN) patches were calculated for each test frame. TP and TN values indicate the number of patches that were truly classified as patches with markers and patches excluding markers (including other objects other than radio-opaque markers), respectively. Different criteria including sensitivity, specificity and precision were used to assess the performance of different classifiers.

The results are illustrated in Figure 2 for the first test data set with the four classifiers: KNN, NB, SVM and LDA. Figure 2a shows the original input frame, stent and catheter
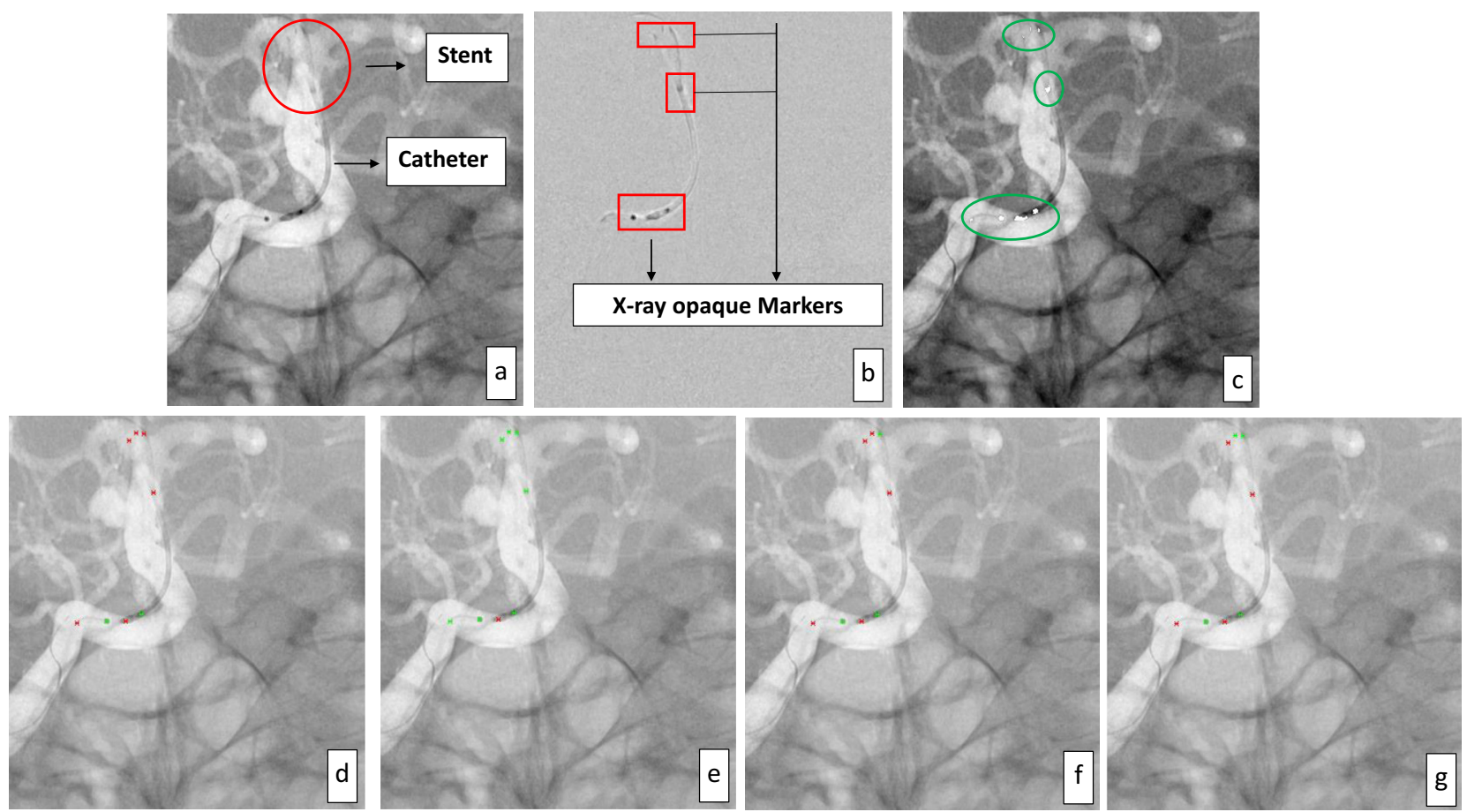

Figure 2: The results of classifications for the first test dataset, a. Original input image, b. pre-processed image, c. Detected areas after thresholding as possible marker candidates, Classification result by d. KNN, e. NB, f. SVM, g. LDA. 


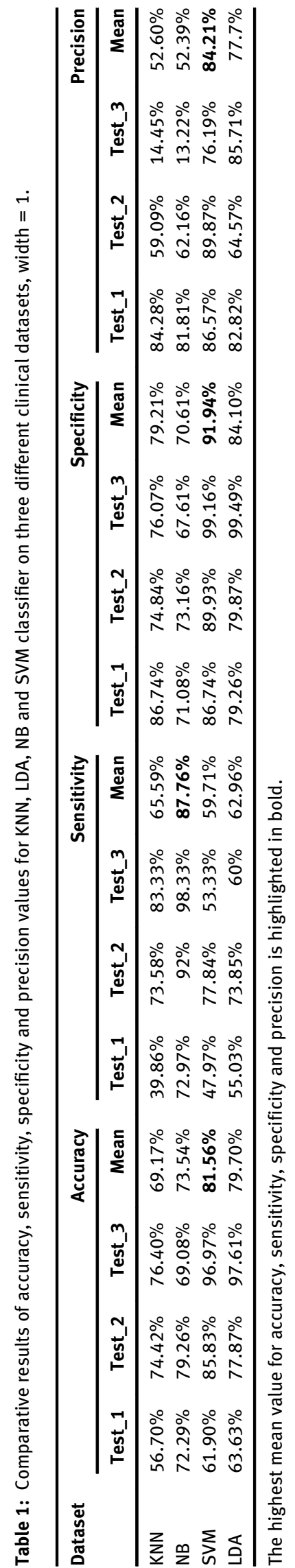

are specified on the image. Figure $2 \mathrm{~b}$ is a pre-processed frame that is used for thresholding. X-ray opaque markers can be better visualized on the pre-processed image. Figure 2c, shows the candidate regions for markers that are surrounded by a green circle and are shown as white regions within the image. Finally, Figure $2(d-f)$ shows prediction results for KNN, NB, SVM and LDA classifier, respectively. Regions marked with green and red stars illustrate predicted marker locations and predicted nonmarker areas, respectively. In this specific frame, KNN predicts two markers truly, while it misses 5 other markers. NB has the best performance for this specific frame by $6 \mathrm{TP}$, $1 \mathrm{FN}$ and $1 \mathrm{FP}$. SVM predicts 3 markers truly while it misclassified 4 other patches (4 FN). LDA also misclassified three marker patches.

The performance of classifiers on 3 test sequences was compared using different criteria (See Table 1). Based on the accuracy results, the NB classifier performs the best on the first test sequence with $72.29 \%$. In the second test sequence, SVM has the highest accuracy of $85.83 \%$. In the last sequence, LDA and SVM have almost the same accuracy value with 97.61 and $96.97 \%$, respectively. Based on the sensitivity value for different classifiers, it concluded that the NB classifier has a lower number of false negatives. NB has the highest mean sensitivity value with $87.76 \%$ and in general based on accuracy, specificity and precision SVM works the best and its average values are 81.56, 91.94 and $84.21 \%$, respectively.

\section{Conclusion}

Markers are very small and hardly visible in 2D X-ray fluoroscopy and our proposed approach can be used to assist clinicians during the intervention with the stent location in 2D by highlighting the detected markers on $\mathrm{X}$-ray images. This eventually leads to faster procedures with less dose exposure to both patients and clinicians. Furthermore, detected markers may be helpful in improving stent visibility in 2D. The adaptive thresholding method used in this study can detect even very small and hardly visible markers, however, to detect all possible candidates, it still depends on the specified sensitivity. Increasing the sensitivity will increase the number of false positives that have to be ruled out in the classification step. This emphasizes the importance of the classification step. Different classifiers including KNN, NB, SVM and LDA were used as a binary classifier to discriminate between nonmarker areas and marker regions. Based on the evaluations, SVM outperforms other classifiers in terms of accuracy, specificity and precision. The highest specificity for 
SVM with $91.94 \%$ was obtained at the cost of low sensitivity. Furthermore, based on the obtained accuracy values for different classifiers, it can be concluded that the performance of the classifiers depends on the dataset. Possible improvements include increasing the number of training patches and increasing features and employing crossvalidation to improve the classifiers' generalizability. Since stent and catheter markers are different in terms of size and shape, employing multi-class classification and considering different types of markers may improve the performance of the classifiers.

Author contributions: All authors have accepted responsibility for the entire content of this manuscript and approved its submission.

Research funding: This work is partly funded by the German Research Foundation (SA3461/2-1) and the Federal Ministry of Education and Research within the Forschungscampus STIMULATE (13GW0095A) as well as partly conducted within the International Graduate School MEMoRIAL at Otto von Guericke University (OVGU) Magdeburg, Germany, which is kindly supported by the European Structural and Investment Funds (ESF) under the programme "SachsenAnhalt WISSENSCHAFT Internationalisierung" (project no. ZS/2016/08/80646).

Conflict of interest: Authors state no conflict of interest. Informed consent: Informed consent is not applicable. Ethical approval: The conducted research is not related to either human or animals use.

\section{References}

1. Reiml S, Pfister M, Toth D, Maier A, Hoffmann M, Kowarschik M, et al. Automatic detection of stent graft markers in $2 \mathrm{~d}$ fluoroscopy images. In: Joint MICCAI workshops on CVII-STENT; 2015.

2. Movassaghi B, Schaefer D, Grass M, Rasche V, Wink O, Garcia JA, et al. $3 \mathrm{~d}$ reconstruction of coronary stents in vivo based on motion compensated $\mathrm{x}$-ray angiograms. In: International conference on medical image computing and computer-assisted intervention: Springer; 2006:177-84 pp.

3. Bismuth V, Vaillant R, Funck F, Guillard N, Najman L. A comprehensive study of stent visualization enhancement in $x$-ray images by image processing means. Med Image Anal 2011;15: 565-76.

4. Wang Y, Chen T, Wang P, Rohkohl C, Comaniciu D. Automatic localization of balloon markers and guidewire in rotational fluoroscopy with application to $3 \mathrm{~d}$ stent reconstruction. In: European conference on computer vision: Springer; 2012:428-41 pp.

5. Schoonenberg G, Lelong P, Florent R, Wink O, ter Haar Romeny B. The effect of automated marker detection on in vivo volumetric stent reconstruction. In: International conference on medical image computing and computer-assisted intervention: Springer; 2008:87-94 pp.

6. Lu X, Chen T, Comaniciu D. Robust discriminative wire structure modeling with application to stent enhancement in fluoroscopy. In: CVPR 2011: IEEE; 2011:1121-7 pp.

7. Chabi N, Beuing O, Preim B, Saalfeld S. Comparison of background removal approaches in x-ray fluoroscopy for detection of cerebral stent markers. In: 18. Jahrestagung der Deutschen Gesellschaft für Computer- und Roboterassistierte Chirurgie e.V; 2019:122-7 pp.

8. Bradley D, Roth G. Adaptive thresholding using the integral image. J Graph Tool 2007;12:13-21.

9. Kroon DJ, Schrijver M. Hessian based frangi vesselness filter: MATLAB File Exchange; 2009:24409. 